\title{
Statistical Estimation of Ergodic Markov Chain Kernel over Discrete State Space
}

\author{
Geoffrey Wolfer \\ geo.wolfer@gmail.com
}

\author{
Aryeh Kontorovich \\ karyeh@cs.bgu.ac.il
}

August 14, 2020

\begin{abstract}
We investigate the statistical complexity of estimating the parameters of a discrete-state Markov chain kernel from a single long sequence of state observations. In the finite case, we characterize (modulo logarithmic factors) the minimax sample complexity of estimation with respect to the operator infinity norm, while in the countably infinite case, we analyze the problem with respect to a natural entry-wise norm derived from total variation. We show that in both cases, the sample complexity is governed by the mixing properties of the unknown chain, for which, in the finite-state case, there are known finite-sample estimators with fully empirical confidence intervals.
\end{abstract}

\section{Introduction}

Approximately recovering the parameters of a discrete distribution is a classical problem in computer science and statistics (see, e.g., Han et al. [2015], Kamath et al. [2015], Orlitsky and Suresh [2015] and the references therein). Total variation (TV) is a natural and wellmotivated choice of approximation metric [Devroye and Lugosi, 2001], and the two metrics we use throughout the paper will be derived from TV. The minimax sample complexity for obtaining an $\varepsilon$-approximation to the unknown distribution in TV (but see Waggoner [2015] for results on other $\ell_{p}$ norms) is well-known to be of the order of $d / \varepsilon^{2}$, where $d$ is the support size (see, e.g., Anthony and Bartlett [1999], Kontorovich and Pinelis [2019]).

This paper deals with estimating the transition probability kernel of a discrete state timehomogeneous Markov chain in the minimax setting. The Markov case is much less wellunderstood than the iid one. The main additional complexity introduced by the Markov case on top of the iid one is that the sample complexity involves not only the number of states and the precision parameter $\varepsilon$, but also the chain's mixing properties.

Our contributions. In the finite-state case, we compute, up to logarithmic factors, (apparently the first, in any metric) high-probability minimax sample complexity for the estimation problem in the Markovian setting, which seeks to recover, from a single long run of an unknown Markov chain, the values of its transition matrix up to a tolerance of $\varepsilon$ in the $\|\cdot\|_{\infty}$ operator norm. We obtain upper and lower bounds on the sample complexity (sequence length) in terms of $\varepsilon$, the number of states, the stationary distribution, and mixing time of the Markov chain. 
In the countably infinite case, for a natural class of chains and with respect to an entry-wise metric derived from TV, we derive an upper bound on the sample complexity that depends in a delicate way on some measure of complexity of the kernel, precision $\varepsilon$ and mixing time, and provide sufficient conditions on the kernel and initial distribution to obtain convergence guarantees.

\section{Definitions and notation}

We denote by $\Omega$ the state space of the Markov chain and by $m$ the size of the sample received by the estimation procedure. The simplex of all distributions over $\Omega$ will be denoted by $\Delta_{\Omega}$, and the set of all Markov kernels by $\mathcal{M}_{\Omega}$. For $|\Omega|<\infty$, we put $d:=|\Omega|$ and $[d]=$ $\Omega=\{1,2, \ldots, d\}$. For $\mu \in \Delta_{\Omega}$, we will write either $\mu(i)$ or $\mu_{i}$, as dictated by esthetics and convenience. All vectors are rows unless indicated otherwise. We use the standard total variation norm, which, up to a convention-dependent factor of 2 , corresponds to the $\ell_{1}$ norm: $2\|\boldsymbol{x}\|_{\mathrm{TV}}=\|\boldsymbol{x}\|_{1}=\sum_{i \in \Omega}\left|x_{i}\right|$. We assume familiarity with basic Markov chain concepts (see, e.g., Kemeny and Snell [1976], Levin et al. [2009]). A time-homogeneous Markov chain $(\boldsymbol{M}, \boldsymbol{\mu})$ on state space $\Omega$ is specified by an initial distribution $\boldsymbol{\mu} \in \Delta_{\Omega}$ and a kernel $\boldsymbol{M} \in \mathcal{M}_{\Omega}$ in the usual way: $\left(X_{1}, \ldots, X_{m}\right) \sim(\boldsymbol{M}, \boldsymbol{\mu})$ means that

$$
\mathbb{P}\left(\left(X_{1}, \ldots, X_{m}\right)=\left(x_{1}, \ldots, x_{m}\right)\right)=\boldsymbol{\mu}\left(x_{1}\right) \prod_{t=1}^{m-1} \boldsymbol{M}\left(x_{t}, x_{t+1}\right)
$$

We write $\mathbb{P}_{\boldsymbol{M}, \mu}(\cdot)$ to denote probabilities over sequences induced by the Markov chain $(\boldsymbol{M}, \boldsymbol{\mu})$, and omit one or both subscripts when clear from context. We say that $\pi$ is a stationary distribution for $\boldsymbol{M}$ if $\pi \boldsymbol{M}=\pi$, and that the Markov chain $(\boldsymbol{M}, \boldsymbol{\mu})$ is stationary if $\boldsymbol{\mu}=\pi$. We will assume the chain to be irreducible and positive recurrent. Namely, $\boldsymbol{M}$ consists of a single communicating class, and defining the return time of state $i$ as $T_{i}=\min \left\{t \geq 0: X_{t}=i\right\}$, we have that for any state $i \in \Omega, \mathbb{E}\left[T_{i}\right]<\infty$. This is sufficient to guarantee existence of a stationary $\pi$. We will further restrict our analysis to geometrically ergodic Markov chains to enable spectral methods.

Definition 2.1 (Geometric ergodicity, Roberts et al. [1997]) The chain $(\boldsymbol{M}, \boldsymbol{\mu})$ with stationary distribution $\pi$ is geometrically ergodic if there is $a \rho \in(0,1)$ and for all $i \in \Omega$ there is a $C_{i} \in \mathbb{R}_{+}$such that

$$
\left\|\boldsymbol{M}^{t}(i, \cdot)-\pi\right\|_{\mathrm{TV}} \leq C_{i} \rho^{t}, \quad t \in \mathbb{N} .
$$

Any chain that satisfies all the above properties will henceforth simply be called ergodic, and all chains mentioned in this work will be assumed ergodic unless stated otherwise. If $\boldsymbol{M}$ is ergodic with stationary distribution $\pi$, then $\pi$ is necessarily unique. To any Markov chain $(\boldsymbol{M}, \boldsymbol{\mu})$, we associate the following measure of non-stationarity

$$
\|\boldsymbol{\mu} / \boldsymbol{\pi}\|_{2, \pi}^{2} \doteq \sum_{i \in \Omega} \boldsymbol{\mu}(i)^{2} / \pi(i) \in[1, \infty]
$$

where the $\|\cdot\|_{2, \pi}$ norm is induced by the inner product in the Hilbert space $\ell_{2}(\pi)$ [Levin et al., 2009 , Chapter 12]. When $|\Omega|<\infty$, we can define the minimum stationary probability by

$$
\pi_{\star} \doteq \min _{i \in \Omega} \pi(i)
$$


In this case, by ergodicity $\pi_{\star}>0$ and $\|\mu / \pi\|_{2, \pi}^{2} \leq \frac{1}{\pi_{\star}}<\infty$. The mixing time of an ergodic $M$ is defined by

$$
t_{\mathrm{mix}} \doteq \inf \left\{t \geq 1: \sup _{\boldsymbol{\mu} \in \Delta_{\Omega}}\left\|\boldsymbol{\mu} \boldsymbol{M}^{t}-\pi\right\|_{\mathrm{TV}}<\frac{1}{4}\right\} .
$$

We define $Q \doteq \operatorname{diag}(\pi) M$ as the matrix $Q(i, j)=\mathbb{P}_{\pi}\left(X_{t}=i, X_{t+1}=j\right)$. A chain $\boldsymbol{M} \in$ $\mathcal{M}_{\Omega}$ is said to be reversible if $Q^{\top}=Q$. The eigenvalues of an ergodic and reversible $M$ lie in $(-1,1]$, and thus may be ordered (counting multiplicities): $1=\lambda_{1}>\lambda_{2} \geq \ldots \geq \lambda_{d}>-1$. The spectral gap and absolute spectral gap of a reversible chain are defined, respectively, by

$$
\gamma \doteq 1-\lambda_{2} \text { and } \gamma_{\star} \doteq 1-\max \left\{\lambda_{2},\left|\lambda_{d}\right|\right\}
$$

Paulin [2015] generalizes the multiplicative reversiblization approach of Fill [1991] by defining the pseudo-spectral gap

$$
\gamma_{\mathrm{ps}} \doteq \max _{k \geq 1}\left\{\gamma\left(\left(\boldsymbol{M}^{\dagger}\right)^{k} \boldsymbol{M}^{k}\right) / k\right\}
$$

where $\boldsymbol{M}^{\dagger}$ is the time reversal of $\boldsymbol{M}$ - the adjoint of $\boldsymbol{M}$ under $\ell_{2}(\pi)-$ given by $\boldsymbol{M}^{\dagger}(i, j) \doteq$ $\pi(j) \boldsymbol{M}(j, i) / \boldsymbol{\pi}(i)$.

For a linear operator $A: \Omega \rightarrow \Omega$,

$$
\|A\|_{\infty}=\sup _{i \in \Omega} \sum_{j \in \Omega}|A(i, j)|
$$

is the operator norm induced by $\ell_{\infty}$ [Horn and Johnson, 1985]. We also define the following entry-wise norm

$$
\|A\| \doteq \sum_{(i, j) \in \Omega^{2}}|A(i, j)|
$$

The norms in (2.6) and (2.7) induce our two notions of distance between Markov kernels $\boldsymbol{M}, \boldsymbol{M}^{\prime}$ with respective stationary distributions $\pi$ and $\pi^{\prime}$ :

$$
\left\|M-M^{\prime}\right\|_{\infty} \text { and }\left\|Q-Q^{\prime}\right\| .
$$

For any $\boldsymbol{M} \in \mathcal{M}_{\Omega}$, define its Dobrushin contraction coefficient

$$
\kappa(\boldsymbol{M}) \doteq \max _{(i, j) \in \Omega^{2}}\|\boldsymbol{M}(i, \cdot)-\boldsymbol{M}(j, \cdot)\|_{\mathrm{TV}} ;
$$

this quantity is also associated with Döblin's name. The term "contraction" refers to the property

$$
\left\|\left(\boldsymbol{\mu}-\boldsymbol{\mu}^{\prime}\right) \boldsymbol{M}\right\|_{\mathrm{TV}} \leq \kappa(\boldsymbol{M})\left\|\boldsymbol{\mu}-\boldsymbol{\mu}^{\prime}\right\|_{\mathrm{TV}}, \quad\left(\boldsymbol{\mu}, \boldsymbol{\mu}^{\prime}\right) \in \Delta_{\Omega^{\prime}}^{2}
$$

which was observed by Markov [1906, §5] (see Kontorovich and Ramanan [2008, Lemma A.2] for an elementary proof).

\section{Main results}

In Section 3.1 we formally state the minimax results for the finite state setting, and then exhibit our results for the countably infinite case in Section 3.2. 


\subsection{Estimation with respect to $\|\cdot\|_{\infty}$ for finite $\Omega$}

Theorem 3.1 (Sample complexity upper bound w.r.t $\|\cdot\|_{\infty}$ when $|\Omega|<\infty$ ) Let $\varepsilon \in(0,2)$, $\delta \in(0,1)$, and let $\boldsymbol{X}=\left(X_{1}, \ldots, X_{m}\right) \sim(\boldsymbol{M}, \boldsymbol{\mu})$, $\boldsymbol{M}$ ergodic with stationary distribution $\pi$. Then an estimator $\widehat{\boldsymbol{M}}: \Omega^{m} \rightarrow \mathcal{M}_{\Omega}$ exists such that whenever

$$
m \geq c \max \left\{\frac{1}{\varepsilon^{2} \pi_{\star}} \max \left\{d, \ln \frac{1}{\varepsilon \delta}\right\}, \frac{1}{\gamma_{\mathrm{ps}} \pi_{\star}} \ln \frac{d\|\mu / \pi\|_{2, \pi}}{\delta}\right\}
$$

we have, with probability at least $1-\delta$,

$$
\|M-\widehat{M}\|_{\infty}<\varepsilon,
$$

where $c$ is a universal constant, $d=|\Omega|, \gamma_{\mathrm{ps}}$ is the pseudo-spectral gap (2.5), $\pi_{\star}$ the minimum stationary probability (2.2), and $\|\boldsymbol{\mu} / \boldsymbol{\pi}\|_{2, \pi}^{2} \leq 1 / \pi_{\star}$ is defined in (2.1).

Although the sample complexity depends on the spectral quantity $\gamma_{\mathrm{ps}}$, and minimal stationary probability $\pi_{\star}$ of the unknown chain, these can be efficiently estimated with finite-sample data-dependent confidence intervals from a single trajectory [Hsu et al., 2019, Wolfer and Kontorovich, 2019b]. Moreover, even though the upper bound formally depends on the unknown (and, in our one-trajectory setting, impossible to estimate) initial distribution $\mu$, we note that ( $i$ ) this dependence is only logarithmic and (ii) an upper bound on $\|\mu / \pi\|_{2, \pi}^{2}$ in terms of $\pi_{\star}$ is easily provided.

Remark 3.1 This upper bound is superior to the one given at Wolfer and Kontorovich [2019a, Theorem 1], shaving a multiplicative factor of $\ln d$ off the first term, except in the extremely high precision regime where $\ln \frac{1}{\varepsilon} \geq d \ln d$.

Theorem 3.2 (Sample complexity lower bound w.r.t $\|\cdot\|_{\infty}$ when $|\Omega|<\infty$ ) For every $\varepsilon \in$ $(0,1 / 32), \gamma_{\mathrm{ps}} \in(0,1 / 8), d=6 k \geq 12$, and every estimation procedure, there exists a $d$ state Markov chain $\boldsymbol{M}$ with pseudo-spectral gap $\gamma_{\mathrm{ps}}$ and stationary distribution $\pi$ such that the estimation procedure must require a sequence $\boldsymbol{X}=\left(X_{1}, \ldots, X_{m}\right)$ drawn from the unknown $M$ of length at least

$$
m \geq c \max \left\{\frac{d}{\varepsilon^{2} \pi_{\star}}, \frac{d \ln d}{\gamma_{\mathrm{ps}}}\right\}
$$

where c is a universal constant, to ensure $\|\boldsymbol{M}-\widehat{\boldsymbol{M}}\|_{\infty}<\varepsilon$ with probability greater than $9 / 10$, and where $d, \gamma_{\mathrm{ps}}, \pi_{\star}$ are as in Theorem 3.1.

The proof of Theorem 3.2 actually yields a bit more than claimed in the statement. For any $\pi_{\star} \in(0,1 / d]$, a Markov chain $\boldsymbol{M}$ can be constructed that achieves the $\frac{d}{\varepsilon^{2} \pi_{\star}}$ component of the bound. Additionally, the $\frac{d}{\gamma_{\mathrm{ps}}}$ component is achievable by a class of reversible Markov chains with spectral gap $\gamma \leq \gamma_{\mathrm{ps}} \leq 2 \gamma$, and uniform stationary distribution - for which $\pi_{\star}=1 / d$ — exhibiting tightness of the obtained bound.

The form of the lower bound indicates that in some regimes, estimating the pseudo-spectral gap up to constant multiplicative error, which requires $\frac{d}{\gamma_{p s}}$ [Hsu et al., 2019, Wolfer and Kontorovich, 2019b], is as difficult as estimating the entire transition matrix (for our choice of metric $\|\cdot\|_{\infty}$ ). We stress that our procedure and guarantees only require ergodicity (and not, say, reversibility) to work. 


\subsection{Results for estimation with respect to $|\|\cdot \mid\|$}

Over an infinite space, $\pi_{\star}=0$ conveys no information, which motivates an alternative notion of distance. For a chain $\boldsymbol{M}$, the kernel of doublet frequencies $\boldsymbol{Q}=\operatorname{diag}(\boldsymbol{\pi}) \boldsymbol{M}$ encodes all information about an ergodic chain [Vidyasagar, 2014], and for two such operators, $Q$ and $Q^{\prime}$ it is the case that

$$
Q=Q^{\prime} \Longrightarrow M=M^{\prime}
$$

Further, it is easily verified that

$$
\left\|Q-Q^{\prime}\right\|=2\left\|Q-Q^{\prime}\right\|_{\mathrm{TV}}
$$

where we see $Q$ and $Q^{\prime}$ as distributions over $\Omega \times \Omega$.

Remark 3.2 The loss of our estimation problem is distinct from the one considered in Hao et al. [2018], which weights the state-wise expected loss with respect to the stationary distribution of the chain, and also allows for sample bounds independent of $\pi_{\star}$.

Theorem 3.3 Let $\varepsilon \in(0,2), \delta \in(0,1)$, and $\boldsymbol{X}=\left(X_{1}, \ldots, X_{m}\right) \sim(\boldsymbol{M}, \boldsymbol{\mu})$, $\boldsymbol{M}$ ergodic with stationary distribution $\pi$, and write $Q=\operatorname{diag}(\pi) M$. There exists an estimator $\widehat{Q}: \Omega^{m} \rightarrow$ $\Delta_{\Omega \times \Omega}$ such that for

$$
m \geq c \frac{t_{\text {mix }}}{\varepsilon^{2}} \max \left\{\|Q\|_{1 / 2}, \ln \left(\frac{\|\mu / \pi\|_{2, \pi}}{\delta}\right)\right\},
$$

we have $\|\widehat{Q}-Q\| \mid<\varepsilon$ with probability at least $1-\delta$, where

$$
\|Q\|_{1 / 2} \doteq\left(\sum_{(i, j) \in \Omega^{2}} \sqrt{Q(i, j)}\right)^{2},
$$

c is a universal constant, $t_{\mathrm{mix}}$ is defined at (2.3), and $\|\boldsymbol{\mu} / \boldsymbol{\pi}\|_{2, \pi}$ is defined in (2.1).

Remark 3.3 Necessary conditions for the upper bound to be non-vacuous are that both $\|\mid Q\| \|_{1 / 2}<$ $\infty$ and $\|\boldsymbol{\mu} / \boldsymbol{\pi}\|_{2, \pi}<\infty$. Importantly, $\|\boldsymbol{\mu} / \boldsymbol{\pi}\|_{2, \pi}<\infty$ implies but is not implied by $\boldsymbol{\mu} \ll \pi$; take, e.g., $\boldsymbol{\mu}(i) \propto \frac{1}{i^{2}}$ and $\pi(i) \propto \frac{1}{i^{4}}$. Notice that in the special case where $|\Omega|=d<\infty$, we

have $\|Q Q\|_{1 / 2} \leq d^{2}$ and the bound reduces to $t_{\operatorname{mix}} \frac{d^{2}}{\varepsilon^{2}}$ (up to logarithmic factors). The mixing time $t_{\mathrm{mix}}$, unknown a priori, can be estimated with finite-sample empirical intervals [Wolfer, 2020].

\section{Overview of techniques}

\subsection{Estimating with respect to the $\|\cdot\|_{\infty}$ norm when $|\Omega|<\infty$}

The upper bound for the estimation problem in Theorem 3.1 is achieved by a (mildly smoothed) natural estimator defined at the beginning of Section 6.1. If the stationary distribution is bounded away from 0 , the chain will visit each state a constant fraction of the total sequence length. Exponential concentration (controlled by the spectral gap) provides high-probability 
confidence intervals about the expectations. A technical complication is that the empirical distribution of the transitions out of a state $i$, conditional on the number of visits $N_{i}$ to that state, is not binomial but actually rather complicated - this is due to the fact that the sequence length is fixed and so a large value of $N_{i}$ "crowds out" other observations. We overcome this by simulating a trajectory from the Markov chain with an array of independent random variables, as described in Billingsley [1961, p.19]. The factor $\|\boldsymbol{\mu} / \pi\|_{2, \pi}$ in the bounds quantifies the price one pays for not assuming (as we do not) stationarity of the unknown Markov chain.

Our chief technical contribution is in establishing the sample complexity lower bounds for the finite space estimation problem. We do this by constructing two distinct lower bounds.

The lower bound of $\frac{d \ln d}{\gamma_{\mathrm{ps}}}$ is derived by a covering argument and a classical reduction scheme to a collection of testing problems using a class of reversible Markov chains we construct, with a carefully controlled pseudo-spectral gap. ${ }^{1}$ The latter can be upper and lower bounded up to universal constants in three key steps. First, we leverage the block structure of the transition matrix of the non-perturbed member of the family to compute its entire spectrum explicitly (Lemma 6.5), and deduce its absolute spectral gap. We then extend the bound to other members of the family, using Markov chain comparison techniques, going through a well known variational definition of the spectral gap. Finally, we conclude by showing that the pseudo-spectral and spectral gap are within a factor of 2 for our class of symmetric Markov chains.

The lower bound of $\frac{d}{\varepsilon^{2} \pi_{\star}}$ is based on the observation that estimating the whole kernel is at least as hard as estimating the conditional distribution a single state. From here, we construct a class of matrices where one state is both hard to reach and difficult to estimate, by constructing mixture of indistinguishable distributions for that particular state, indexed by a large subset of the binary hypercube. We express the statistical distance between words of length $m$ distributed according to different matrices of this class in terms of $\pi_{\star}$ and the KL divergence between the conditional distributions of the hard-to-reach state, by taking advantage of the structure of the class, and invoke an argument from Tsybakov to conclude ours.

\subsection{Estimating with respect to the $\||\cdot|||$ metric}

The extension to a countably infinite setting requires an alternative notion of distance between chains. The proof then introduces the natural counting estimator $\widehat{Q}(i, j)=\frac{N_{i j}}{m-1}$ of transitions from $i$ to $j$, and starts by controlling the error in expectation. It reduces the problem to the study of the variance of the random variable $N_{i j}$, which is achieved by constructing another Markov chain with approximately the same mixing time, and invoking known results from Paulin [2015] for the variance of sums of functions under the Markovian setting. The result is then obtained by controlling the fluctuations around this expectation by a bounded differences argument.

\section{Related work}

Our Markov chain statistical estimation setup is a natural extension of the PAC distribution learning model of Kearns et al. [1994]. Despite the plethora of literature on estimating Markov transition matrices, (see, e.g., Billingsley [1961], Craig and Sendi [2002], Welton and Ades [2005]) we were not able to locate any rigorous finite-sample PAC-type results.

\footnotetext{
${ }^{1}$ The family of chains used in the lower bound of Hsu et al. [2019] does not suffice for our purposes; a considerably richer family is needed (see Remark 6.3).
} 
The minimax problem has recently received some attention, and Hao et al. [2018] have, in parallel to us, shown the first minimax bounds, in expectation, for the problem of estimating the transition matrix $\boldsymbol{M}$ of a Markov chain under a certain class of divergences. The authors consider the case where $\min _{i, j} \boldsymbol{M}(i, j) \geq \alpha>0$, essentially showing that for some family of smooth $f$-divergences, the expected risk is of the order of $\frac{d f^{\prime \prime}(1)}{m \pi_{\star}}$. The metric used in this paper is based on TV, which corresponds to the $f$-divergence induced by $f(t)=\frac{1}{2}|t-1|$, which is not differentiable at $t=1$. The results of Hao et al. and the present paper are complementary and not directly comparable. We do note that $(i)$ their guarantees are in expectation rather than with high-confidence, (ii) our TV-based metric is not covered by their smooth $f$-divergence family, and most important (iii) their notion of mixing is related to contraction as opposed to the spectral gap. In particular the $\alpha$-minoration assumption implies (but is not implied by) a bound of $\kappa \leq 1-d \alpha$ on the Dobrushin contraction coefficient (defined in (2.8); see Kontorovich [2007, Lemma 2.2.2] for the latter claim). Thus, the family of $\alpha$-minorized Markov chains is strictly contained in the family of contracting chains, which in turn is a strict subset of the ergodic chains we consider.

This paper is based on the conference version of Wolfer and Kontorovich [2019a] together with an extension to countably infinite spaces at Section 3.2. Another key improvement over the extended abstract is in the proof of Theorem 3.2. While the series of lemmas Wolfer and Kontorovich [2019a, Lemma 8, Lemma 9, Lemma 11] showed that it is possible to control the pseudo-spectral gap of our special family of chains via Cheeger's inequality combined with a contraction-based argument, this technique relied on heavy computations to bound the Dobrushin coefficient of the two-step transition matrix. Moreover the proof for the extension to all members of the class was only sketched in Wolfer and Kontorovich [2019a, Lemma 9]. In the present manuscript, we switch technique, compute the full spectrum of the unperturbed transition matrix instead, and fully flesh out the proof for the extension to perturbed chains using comparison techniques. Finally, the upper bound at Theorem 3.1 also improves upon Wolfer and Kontorovich [2019a, Theorem 1], by relying on a simulation scheme from Billingsley, instead of martingale techniques.

\section{Proofs}

\subsection{Proof of Theorem 3.1}

Remark 6.1 We thank an anonymous referee for the suggestion (and technique) to improve the logarithmic gap between the upper and lower bounds.

Let $\varepsilon \in(0,2), \delta \in(0,1)$, let $\boldsymbol{M}$ be a $d$-state ergodic Markov kernel with stationary distribution $\pi$, and first consider the stationary case $X_{1}, \ldots, X_{m} \sim(\boldsymbol{M}, \pi)$. We define the natural counting random variables

$$
N_{i} \doteq \sum_{t=1}^{m-1} \mathbf{1}\left\{X_{t}=i\right\}, \quad N_{i j} \doteq \sum_{t=1}^{m-1} \mathbf{1}\left\{X_{t}=i, X_{t+1}=j\right\}
$$

and the estimator of the kernel will be $\widehat{\boldsymbol{M}}(i, j) \doteq \frac{N_{i j}}{N_{i}}$ when $N_{i} \neq 0$ and $1 / d$ when $N_{i}=0$. We decompose the error probability of the estimation procedure, while choosing an arbitrary value 
$n_{i} \in \mathbb{N}$ for the desired number of visits to each state $i \in[d]$,

$$
\begin{aligned}
\mathbb{P}_{\pi}\left(\|\boldsymbol{M}-\widehat{\boldsymbol{M}}\|_{\infty}>\varepsilon\right) & \leq \sum_{i=1}^{d} \sum_{n=n_{i}}^{3 n_{i}} \mathbb{P}_{\pi}\left(\|\widehat{\boldsymbol{M}}(i, \cdot)-\boldsymbol{M}(i, \cdot)\|_{1}>\varepsilon \text { and } N_{i}=n\right) \\
& +\mathbb{P}_{\pi}\left(\left\{\exists i \in[d]: N_{i} \notin\left[n_{i}, 3 n_{i}\right]\right\}\right) .
\end{aligned}
$$

We simulate a trajectory from $\boldsymbol{M}$ with a collection of independent samples using the scheme described in [Billingsley, 1961, p.19], where we define the following infinite array of random variables,

$$
\begin{array}{lllll}
X_{1,1} & X_{1,2} & \cdots & X_{1, t} & \cdots \\
X_{2,1} & X_{2,2} & \cdots & X_{2, t} & \cdots \\
\cdots & \cdots & \cdots & \cdots & \cdots \\
X_{d, 1} & X_{d, 2} & \cdots & X_{d, t} & \cdots
\end{array}
$$

such that $\forall(i, j, t) \in[d]^{2} \times \mathbb{N}, \mathbb{P}\left(X_{i, t}=j\right)=\boldsymbol{M}(i, j)$, and the sampling procedure is as follows. Start by drawing $\tilde{X}_{1} \sim \pi$. $\tilde{X}_{2}$ is then defined to be $X_{\tilde{X}_{1}, 1}$, the first element of the $\tilde{X}_{1}$ th row. The process then continues inductively recording random variables from left to right in their corresponding rows, such that if $\tilde{X}_{1}, \tilde{X}_{2}, \ldots, \tilde{X}_{t}$ have been defined, then $\tilde{X}_{t+1} \doteq$ $X_{\tilde{X}_{t}, \tilde{N}_{\tilde{X}_{t}}^{(t)}+1}$ where $\tilde{N}_{i}^{(t)} \doteq \sum_{s=1}^{t} \mathbf{1}\left\{\tilde{X}_{s}=i\right\}$, and for convenience, $\tilde{N}_{i} \doteq \tilde{N}_{i}^{(m-1)}$. Observe that $X_{1}, X_{2}, \ldots$, and $\tilde{X}_{1}, \tilde{X}_{2}, \ldots$ are identically distributed. Then, writing

$$
\widetilde{\boldsymbol{M}}(i, \cdot) \doteq \frac{1}{\tilde{N}_{i}} \sum_{j=1}^{d} \sum_{t=1}^{m-1} \mathbf{1}\left\{\tilde{X}_{t}=i, \tilde{X}_{t+1}=j\right\} \boldsymbol{e}_{j},
$$

we have

$\mathbb{P}_{\pi}\left(\|\widehat{\boldsymbol{M}}(i, \cdot)-\boldsymbol{M}(i, \cdot)\|_{1}>\varepsilon\right.$ and $\left.N_{i}=n\right)=\mathbb{P}\left(\|\widetilde{\boldsymbol{M}}(i, \cdot)-\boldsymbol{M}(i, \cdot)\|_{1}>\varepsilon\right.$ and $\left.\tilde{N}_{i}=n\right)$.

In the event where $\tilde{N}_{i}=n$,

$$
\begin{aligned}
\widetilde{\boldsymbol{M}}(i, \cdot) & =\frac{1}{n} \sum_{j=1}^{d} \sum_{t=1}^{m-1} \mathbf{1}\left\{\tilde{X}_{t}=i, X_{\tilde{X}_{t}, \tilde{N}_{\tilde{X}_{t}}^{(t)}+1}=j\right\} \boldsymbol{e}_{j} \\
& =\frac{1}{n} \sum_{j=1}^{d} \sum_{t=1}^{n} \mathbf{1}\left\{X_{i, t}=j\right\} \boldsymbol{e}_{j} \\
& \doteq \widetilde{\boldsymbol{M}}_{n}(i, \cdot),
\end{aligned}
$$

where by definition, $X_{i, 1}, X_{i, 2}, \ldots, X_{i, n} \sim \boldsymbol{M}(i, \cdot)^{\otimes n}$, and the problem is reduced to learning a distribution out of $n$ independent samples. Since $\mathbb{E}\left\|\widetilde{\boldsymbol{M}}_{n}(i, \cdot)-\boldsymbol{M}(i, \cdot)\right\|_{1} \leq \sqrt{\frac{d}{n}}$, (see for example Berend and Kontorovich [2013]) and the function $X_{i, 1}, X_{i, 2}, \ldots, X_{i, n} \mapsto$ $\left\|\widetilde{\boldsymbol{M}}_{n}(i, \cdot)-\boldsymbol{M}(i, \cdot)\right\|_{1}$ is $(2 / n)$-Lipschitz, an application of McDiarmid's inequality yields that

$$
\begin{aligned}
\mathbb{P}\left(\|\widetilde{\boldsymbol{M}}(i, \cdot)-\boldsymbol{M}(i, \cdot)\|_{1}>\varepsilon \text { and } \tilde{N}_{i}=n\right) & \leq \mathbb{P}\left(\left\|\widetilde{\boldsymbol{M}}_{n}(i, \cdot)-\boldsymbol{M}(i, \cdot)\right\|_{1}>\varepsilon\right) \\
& \leq \exp \left(-\frac{n}{2} \max \left\{0, \varepsilon-\sqrt{\frac{d}{n}}\right\}^{2}\right) .
\end{aligned}
$$


It follows that

$$
\begin{aligned}
& \sum_{n=n_{i}}^{3 n_{i}} \mathbb{P}_{\pi}\left(\|\widehat{\boldsymbol{M}}(i, \cdot)-\boldsymbol{M}(i, \cdot)\|_{1}>\varepsilon \text { and } N_{i}=n\right) \\
& \stackrel{(i)}{\leq}\left(2 n_{i}+1\right) \exp \left(-\frac{n_{i}}{2} \max \left\{0, \varepsilon-\sqrt{\frac{d}{n_{i}}}\right\}^{2}\right) \\
& \stackrel{(i i)}{\leq}\left(m \pi_{i}+1\right) \exp \left(-c m \pi_{i} \varepsilon^{2}\right),
\end{aligned}
$$

where $c=\frac{(1-1 / \sqrt{2})^{2}}{4},(i)$ stems from a monotonicity argument, and $(i i)$ is by setting $n_{i}=\frac{m \pi_{i}}{2}$, and as long as $m \geq \frac{4 d}{\varepsilon^{2} \pi_{\star}}$. We start by handling the first term of (6.1),

$$
\begin{aligned}
\sum_{i=1}^{d}\left(m \pi_{i}+1\right) \exp \left(-c m \pi_{i} \varepsilon^{2}\right) & \leq \sum_{i=1}^{d} \frac{1}{c \varepsilon^{2}} \exp \left(-c m \pi_{i} \varepsilon^{2} / 2\right)+\sum_{i=1}^{d} \exp \left(-c m \pi_{i} \varepsilon^{2}\right) \\
& \leq \sum_{i=1}^{d} \frac{2}{c \varepsilon^{2}} \exp \left(-c m \pi_{i} \varepsilon^{2} / 2\right) \\
& \leq \frac{2 d}{c \varepsilon^{2}} \exp \left(-c m \pi_{\star} \varepsilon^{2} / 2\right)
\end{aligned}
$$

where we used the fact that $x>0 \Longrightarrow x \exp (-x) \leq \exp (-x / 2)$, and which is smaller than $\delta / 2$ as long as $m \geq \frac{2}{c \pi_{\star} \varepsilon^{2}} \ln \frac{4 d}{c \delta \varepsilon^{2}}$. It remains to control the probability of the bad event where the states are not visited a reasonable amount of time. Invoking Paulin [2015, Theorem 3.10],

$$
\mathbb{P}_{\pi}\left(N_{i} \notin\left[\frac{1}{2} m \pi_{i}, \frac{3}{2} m \pi_{i}\right]\right) \leq \exp \left(-\frac{\gamma_{\mathrm{ps}}\left(\frac{1}{2} m \pi_{i}\right)^{2}}{8\left(m+1 / \gamma_{\mathrm{ps}}\right) \pi_{i}\left(1-\pi_{i}\right)+20 \frac{1}{2} m \pi_{i}}\right) .
$$

Quantifying the price for non-stationarity using Paulin [2015, Proposition 3.14],

$$
\mathbb{P}_{\mu}\left(\|\widehat{M}-M\|_{\infty}>\varepsilon\right) \leq\|\mu / \pi\|_{2, \pi} \sqrt{\mathbb{P}_{\pi}\left(\|\widehat{M}-M\|_{\infty}>\varepsilon\right)}
$$

and combining with (6.3) yields the upper bound.

Remark 6.2 Note that one can derive an upper bound of $\frac{1}{\pi_{\star}} \max \left\{1 / \varepsilon^{2}, 1 / \gamma_{\mathrm{ps}}\right\}$ (up to logarithmic factors) for the problem with respect to the max norm

$$
\|\boldsymbol{M}-\widehat{\boldsymbol{M}}\|_{\mathrm{MAX}}=\max _{(i, j) \in[d]^{2}}|\boldsymbol{M}(i, j)-\widehat{\boldsymbol{M}}(i, j)| .
$$

Similarly, for $p \in[1,2)$, we can derive the more general upper bound (up to logarithmic factors)

$$
\frac{1}{\pi_{\star}} \max \left\{\frac{d^{2 / p-1}}{\varepsilon^{2}}, \frac{1}{\gamma_{\mathrm{ps}}}\right\}
$$

for the problem with respect to the norm $\|\boldsymbol{M}-\widehat{\boldsymbol{M}}\|_{\infty, p} \doteq \max _{i \in[d]}\|\boldsymbol{M}(i, \cdot)-\widehat{\boldsymbol{M}}(i, \cdot)\|_{p}$. 


\subsection{Proof of Theorem 3.3}

Sample $X_{1}, \ldots, X_{m} \sim(\boldsymbol{M}, \boldsymbol{\mu})$ where $\boldsymbol{M}$ is ergodic with stationary distribution $\pi$. We define the estimator $\widehat{Q} \doteq \frac{1}{m-1} N_{i j}$ with $N_{i j} \doteq \sum_{t=1}^{m-1} \mathbf{1}\left\{X_{t}=i, X_{t+1}=j\right\}$. We first focus on the stationary case where $\mu=\pi$.

Bounding the distance in expectation. From Jensen's inequality and stationarity,

$$
\begin{aligned}
\mathbb{E}_{\pi}\|\widehat{Q}-Q\| \| & =\sum_{(i, j) \in \Omega^{2}} \mathbb{E}_{\pi}\left|\frac{N_{i j}}{m-1}-\pi(i) \boldsymbol{M}(i, j)\right| \\
& \leq \frac{1}{m-1} \sum_{(i, j) \in \Omega^{2}} \sqrt{\mathbb{E}_{\pi}\left[\left(N_{i j}-(m-1) \pi(i) \boldsymbol{M}(i, j)\right)^{2}\right]} \\
& =\frac{1}{m-1} \sum_{(i, j) \in \Omega^{2}} \sqrt{\operatorname{Var}_{\pi}\left[N_{i j}\right]}
\end{aligned}
$$

and we are left with controlling a variance term. The next lemma defines a new Markov chain from $X_{1}, \ldots, X_{m}$ with an approximately similar mixing time.

Lemma 6.1 Let $\boldsymbol{X}=\left(X_{1}, \ldots, X_{m}\right) \sim(M, \pi)$ with mixing time $t_{\mathrm{mix}}$ and stationary distribution $\pi$, then $\boldsymbol{Y}=\left(\left(X_{1}, X_{2}\right),\left(X_{2}, X_{3}\right), \ldots,\left(X_{m-1}, X_{m}\right)\right)$ is also a finite state Markov chain with mixing time at most $t_{\mathrm{mix}}+1$, with kernel $\widetilde{\boldsymbol{M}}: \Omega^{2} \times \Omega^{2} \rightarrow[0,1]$ and stationary distribution $\tilde{\pi}$, such that for all $(i, j, k, \ell) \in \Omega^{4}$,

$$
\begin{aligned}
\widetilde{\boldsymbol{M}}((i, j),(k, \ell)) & =\mathbf{1}\{k=j\} \boldsymbol{M}(k, \ell) \\
\tilde{\boldsymbol{\pi}}((i, j)) & =\boldsymbol{Q}(i, j) .
\end{aligned}
$$

Proof: Let $\boldsymbol{X}=\left(X_{1}, \ldots, X_{m}\right) \sim(M, \pi)$ with mixing time $t_{\text {mix }}$ and stationary distribution $\pi$, we first show that $\boldsymbol{Y}=\left(\left(X_{1}, X_{2}\right),\left(X_{2}, X_{3}\right), \ldots,\left(X_{m-1}, X_{m}\right)\right)$ is also a finite state Markov chain. For all $t \in \mathbb{N}$ and $\boldsymbol{y}=\left(y_{1}, \ldots, y_{t-1}\right)$ with $y_{s}=\left(x_{s}, x_{s+1}\right)$ for $s \in[t-2]$, and whenever defined,

$$
\begin{aligned}
& \mathbb{P}\left(Y_{t}=(i, j) \mid Y_{1}=y_{1}, \ldots, Y_{t-1}=y_{t-1}\right) \\
& =\mathbb{P}\left(\left(X_{t}, X_{t+1}\right)=(i, j) \mid X_{1}=x_{1}, \ldots, X_{t-1}=x_{t-1}, X_{t}=x_{t}\right) \\
& =\mathbf{1}\left\{i=x_{t}\right\} \mathbb{P}\left(X_{t+1}=j \mid X_{t}=x_{t}\right)=\mathbf{1}\left\{i=x_{t}\right\} \mathbf{M}(i, j) \\
& =\mathbb{P}\left(Y_{t}=(i, j) \mid Y_{t-1}=y_{t-1}\right),
\end{aligned}
$$

which confirms the Markov property. Additionally, setting $\tilde{\pi}((i, j))=Q(i, j)$,

$$
\begin{aligned}
\sum_{(i, j) \in \Omega^{2}} \tilde{\boldsymbol{\pi}}((i, j)) \widetilde{\boldsymbol{M}}((i, j),(k, \ell)) & =\sum_{(i, j) \in \Omega^{2}} \tilde{\boldsymbol{\pi}}((i, j)) \mathbf{1}\{k=j\} \boldsymbol{M}(k, \ell)=\boldsymbol{M}(k, \ell) \sum_{i \in \Omega} \tilde{\boldsymbol{\pi}}((i, k)) \\
& =\boldsymbol{M}(k, \ell) \sum_{i \in \Omega} \boldsymbol{\pi}(i) \boldsymbol{M}(i, k)=\boldsymbol{M}(k, \ell) \boldsymbol{\pi}(k)=\tilde{\boldsymbol{\pi}}((k, \ell)) .
\end{aligned}
$$

This entails that $\boldsymbol{Y}$ is a Markov chain $(\tilde{\boldsymbol{\pi}}, \widetilde{\boldsymbol{M}})$ over the state space $\Omega \times \Omega$, and stationary distribution $\tilde{\pi}$. Let $t \geq t_{\text {mix }}+1$, and $\tilde{\delta}_{\left(i_{1}, j_{1}\right)}$ the distribution on $\Omega \times \Omega$ that puts mass 1 at $\left(i_{1}, j_{1}\right)$ and 0 everywhere else, then

$$
\left\|\tilde{\delta}_{\left(i_{1}, j_{1}\right)} \widetilde{\boldsymbol{M}}^{t}-\tilde{\boldsymbol{\pi}}\right\|_{\mathrm{TV}}=\sum_{(i, j) \in \Omega^{2}}\left|\tilde{\delta}_{\left(i_{1}, j_{1}\right)} \widetilde{\boldsymbol{M}}^{t}(i, j)-\tilde{\boldsymbol{\pi}}(i, j)\right| .
$$


One one hand,

$$
\begin{aligned}
\tilde{\delta}_{\left(i_{1}, j_{1}\right)} \widetilde{\boldsymbol{M}}^{t}(i, j) & =\mathbb{P}\left(Y_{t+1}=(i, j) \mid Y_{1}=\left(i_{1}, j_{1}\right)\right) \\
& =\mathbb{P}\left(\left(X_{t+1}, X_{t+2}\right)=(i, j) \mid\left(X_{1}, X_{2}\right)=\left(i_{1}, j_{1}\right)\right) \\
& =\mathbb{P}\left(X_{t+2}=j \mid X_{t+1}=i\right) \mathbb{P}\left(X_{t+1}=i \mid X_{2}=j_{1}\right) \\
& =\boldsymbol{M}(i, j) \boldsymbol{M}^{t-1}\left(j_{1}, i\right)
\end{aligned}
$$

so that

$$
\begin{aligned}
\left\|\tilde{\delta}_{\left(i_{1}, j_{1}\right)} \widetilde{\boldsymbol{M}}^{t}-\tilde{\boldsymbol{\pi}}\right\|_{\mathrm{TV}} & =\frac{1}{2} \sum_{(i, j) \in \Omega^{2}}\left|\boldsymbol{M}(i, j) \boldsymbol{M}^{t-1}\left(j_{1}, i\right)-\boldsymbol{M}(i, j) \boldsymbol{\pi}(i)\right| \\
& =\frac{1}{2} \sum_{i \in \Omega}\left|\boldsymbol{M}^{t-1}\left(j_{1}, i\right)-\pi(i)\right|=\left\|\delta_{j_{1}} \boldsymbol{M}^{t-1}-\pi\right\|_{\mathrm{TV}} \leq 1 / 4,
\end{aligned}
$$

by definition of $t_{\mathrm{mix}}$, and by the condition on $t$.

Corollary 6.1 Let $X_{1}, \ldots, X_{m} \sim(\boldsymbol{M}, \boldsymbol{\pi})$ and define the chain $\boldsymbol{Y} \sim(\widetilde{\boldsymbol{M}}, \tilde{\boldsymbol{\pi}})$ from Lemma 6.1, then by Paulin [2015, Theorem 3.2, Proposition 3.4], for $\phi: \Omega^{2} \rightarrow \mathbb{R}^{+}$,

$$
\operatorname{Var}_{\tilde{\pi}}\left[\sum_{t=1}^{m-1} \phi\left(Y_{t}\right)\right] \leq \frac{4 m}{\gamma_{\mathrm{ps}}} \mathbb{V} \mathbf{a r}_{\tilde{\pi}}[\phi] \leq 8 m t_{\mathrm{mix}} \operatorname{Var}_{\tilde{\pi}}[\phi]
$$

and from the fact that

$$
\operatorname{Var}_{\boldsymbol{\pi}}\left[\mathbf{1}\left\{X_{t}=i, X_{t+1}=j\right\}\right]=\boldsymbol{\pi}(i) \boldsymbol{M}(i, j)(1-\boldsymbol{\pi}(i) \boldsymbol{M}(i, j)),
$$

we get the following control on the variance term:

$$
\operatorname{Var}_{\boldsymbol{\pi}}\left[N_{i j}\right] \leq 8 m t_{\text {mix }} \boldsymbol{\pi}(i) \boldsymbol{M}(i, j)(1-\boldsymbol{\pi}(i) \boldsymbol{M}(i, j)) .
$$

As a consequence of Corollary 6.1,

$$
\mathbb{E}_{\pi} \mid\|\widehat{Q}-Q\| \leq 2 \sqrt{\frac{t_{\operatorname{mix}}+1}{m-1}\|Q\| \|_{1 / 2}}, \text { where }\|Q\| \|_{1 / 2} \doteq\left(\sum_{(i, j) \in \Omega^{2}} \sqrt{Q(i, j)}\right)^{2}
$$

and

$$
m \geq 64 \frac{\|Q\| \|_{1 / 2} t_{\text {mix }}}{\varepsilon^{2}} \Longrightarrow \mathbb{E}_{\pi}\|\widehat{Q}-Q\| \mid \leq \varepsilon / 2
$$

Bounding the fluctuations around the expectation. The strategy is to show that the loss is $(4 /(m-1))$-Lipschitz, and simply invoke McDiarmid's inequality for Markov chains [Paulin, 2015, Corollary 2.10]. 
For $x=\left(x_{1}, \ldots, x_{m}\right) \in \mathbb{R}^{m}$, let $x^{(k)}=\left(x_{1}, \ldots, x_{k-1}, x_{k}^{\prime}, x_{k+1}, \ldots, x_{m}\right)$, with $k \in[m]$,

$$
\begin{aligned}
& \|\| \widehat{\boldsymbol{Q}}(\boldsymbol{x})-\boldsymbol{Q}\left\|\left|-\left\|\widehat{\boldsymbol{Q}}\left(\boldsymbol{x}^{\prime}\right)-\boldsymbol{Q}\right\|\right| \mid\right. \\
& =\frac{1}{m-1}\left|\sum_{(i, j) \in \Omega^{2}}\left(\left|n_{i j}-(m-1) \boldsymbol{Q}(i, j)\right|-\left|n_{i j}^{(k)}-(m-1) \boldsymbol{Q}(i, j)\right|\right)\right| \\
& \leq \frac{1}{m-1} \sum_{(i, j) \in \Omega^{2}}|| n_{i j}-(m-1) \boldsymbol{Q}(i, j)|-| n_{i j}^{(k)}-(m-1) \boldsymbol{Q}(i, j)|| \\
& \leq \frac{1}{m-1} \sum_{(i, j) \in \Omega^{2}}\left|n_{i j}-n_{i j}^{(k)}\right|,
\end{aligned}
$$

where we successively invoked the forward and reverse triangle inequality. We then compute

$$
\begin{aligned}
\sum_{(i, j) \in \Omega^{2}}\left|n_{i j}-n_{i j}^{(k)}\right|= & \sum_{(i, j) \in \Omega^{2}} \mid \mathbf{1}\left\{x_{k}=i, x_{k+1}=j\right\}-\mathbf{1}\left\{x_{k}^{(k)}=i, x_{k+1}^{(k)}=j\right\}+ \\
& \mathbf{1}\left\{x_{k-1}=i, x_{k}=j\right\}-\mathbf{1}\left\{x_{k-1}^{(k)}=i, x_{k}^{(k)}=j\right\} \mid \\
\leq & \sum_{(i, j) \in \Omega^{2}}\left(\mathbf{1}\left\{x_{k}=i, x_{k+1}=j\right\}+\mathbf{1}\left\{x_{k}^{\prime}=i, x_{k+1}=j\right\}+\right. \\
& \left.\mathbf{1}\left\{x_{k-1}=i, x_{k}=j\right\}+\mathbf{1}\left\{x_{k-1}=i, x_{k}^{\prime}=j\right\}\right) \leq 4
\end{aligned}
$$

so that \|\|$\widehat{Q}(x)-Q\|\|-\left\|\left|\widehat{Q}\left(x^{\prime}\right)-Q\|\|\right| \leq \frac{4}{m-1}\right.$, and it is then a consequence of McDiarmid's inequality for Markov chains [Paulin, 2015, Corollary 2.10] that

$$
\mathbb{P}_{\pi}\left(\|\| \widehat{\boldsymbol{Q}}(\boldsymbol{X})-\boldsymbol{Q}\left\|\left|-\mathbb{E}_{\pi}\||\widehat{\boldsymbol{Q}}(\boldsymbol{X})-\boldsymbol{Q}\|\||>\varepsilon / 2) \leq 2 \exp \left(-\frac{m \varepsilon^{2}}{c t_{\text {mix }}}\right), c \in \mathbb{R}_{+}\right.\right.\right.
$$

and $m \geq \frac{c t_{\text {mix }}}{\varepsilon^{2}} \ln \left(\frac{2}{\delta}\right) \Longrightarrow \mathbb{P}_{\pi}(\|\| \widehat{Q}-Q\|\|-\pi\|\widehat{Q}(x)-Q\| \| \mid>\varepsilon / 2) \leq \delta$. Finally, we extend the study to non-stationary chains in a straightforward way as for the proof of Theorem 6.1, with Paulin [2015, Proposition 3.10], which yields the final theorem.

\subsection{Proof of Theorem $3.2\left(\right.$ part 1): $\frac{d}{\varepsilon^{2} \pi_{\star}}$}

Recall the definition of KL divergence between two distributions $(\boldsymbol{v}, \boldsymbol{\theta}) \in \Delta_{\Omega}^{2}$, such that $\boldsymbol{\theta} \ll$ $\boldsymbol{\theta}$,

$$
D_{K L}(\boldsymbol{v} \| \boldsymbol{\theta}) \doteq \sum_{i \in \Omega} \boldsymbol{v}(i) \ln \frac{\boldsymbol{v}(i)}{\boldsymbol{\theta}(i)} .
$$

Let $\varepsilon \in(0,1 / 32)$, and $\mathcal{M}_{d, \gamma_{\mathrm{ps}}, \pi_{\star}}$ be the collection of all $d$-state Markov chains whose stationary distribution is minorized by $\pi_{\star}$ and whose pseudo-spectral gap is at least $\gamma_{\mathrm{ps}}$. The quantity we wish to lower bound is the minimax risk for the estimation problem :

$$
\mathcal{R}_{m}=\inf _{\widehat{M}} \sup _{\boldsymbol{M}} \mathbb{P}_{\boldsymbol{M}}\left(\|\boldsymbol{M}-\widehat{\boldsymbol{M}}\|_{\infty}>\varepsilon\right),
$$


where the inf is taken over all estimation procedures $\widehat{\boldsymbol{M}}:[d]^{m} \rightarrow \mathcal{M}_{d}, \boldsymbol{X} \mapsto \widehat{\boldsymbol{M}}(\boldsymbol{X})$ and the sup over $\mathcal{M}_{d, \gamma_{\mathrm{ps}}, \pi_{\star}}$. Suppose for simplicity of the analysis that we consider Markov chains of $d+1$ states instead of $d$, and that $d$ is even. A slight modification of the proofs covers the odd case. We define the following class of Markov chains parametrized by a given distribution $p \in \Delta_{d+1}$, where the conditional distribution defined at each state of the chain is always $p$ with $p_{d+1}=p_{\star}$ and $p_{k}=\frac{1-p_{\star}}{d}$ for $k \in[d]$, with $p_{\star}<\frac{1}{d+2}$, except for state $d+1$, where it is only required that it has a loop of probability $p_{\star}$ to itself.

$$
\mathcal{G}_{p}=\left\{\boldsymbol{M}_{\eta}=\left(\begin{array}{cccc}
p_{1} & \ldots & p_{d} & p_{\star} \\
\vdots & \vdots & \vdots & \vdots \\
p_{1} & \ldots & p_{d} & p_{\star} \\
\eta_{1} & \ldots & \eta_{d} & p_{\star}
\end{array}\right): \boldsymbol{\eta}=\left(\eta_{1}, \ldots, \eta_{d}, p_{\star}\right) \in \Delta_{d+1}\right\} .
$$

Remark: a family of Markov chains very similar to $\mathcal{G}_{p}$ was independently considered by Hao et al. [2018] for proving their lower bound.

It is easy to see that the stationary distribution $\pi$ of an element of $\mathcal{G}_{p}$ indexed by $\eta$ is

$$
\pi_{k}=\frac{\left(1-p_{\star}\right)^{2}}{d}+\eta_{k} p_{\star}, \text { for } k \in[d], \quad \pi_{d+1}=p_{\star} .
$$

For $m \geq 4, \boldsymbol{\eta}=\left(\eta_{1}, \ldots, \eta_{d}, p_{\star}\right) \in \Delta_{d+1}$ and $\left(X_{1}, \ldots, X_{m}\right) \sim\left(\boldsymbol{M}_{\eta}, \boldsymbol{p}\right)$, set $N_{i}=\sum_{t=1}^{m} \mathbf{1}\left\{X_{t}=i\right\}$ the number of visits to the $i$ th state. Focusing on the $(d+1)$ th state, since for $i \in[d+1]$, we have $\boldsymbol{M}_{\eta}(i, d+1)=p_{\star}$, it is immediate that $N_{d+1} \sim \operatorname{Binomial}\left(m, p_{\star}\right)$. Introduce the subset of Markov chains in $\mathcal{G}_{p}$ such that

$$
\eta(\sigma)=\left(\frac{1-p_{\star}+16 \sigma_{1} \varepsilon}{d}, \frac{1-p_{\star}-16 \sigma_{1} \varepsilon}{d}, \ldots, \frac{1-p_{\star}+16 \sigma_{\frac{d}{2}} \varepsilon}{d}, \frac{1-p_{\star}-16 \sigma_{\frac{d}{2}} \varepsilon}{d}, p_{\star}\right),
$$

where $\sigma=\left(\sigma_{1}, \ldots, \sigma_{\frac{d}{2}}\right) \in\{-1,1\}^{\frac{d}{2}}$. Also define $\boldsymbol{M}_{0}$ with $\eta_{0}=\left(\frac{1-p_{\star}}{d}, \ldots, \frac{1-p_{\star}}{d}, p_{\star}\right)$. We start by showing that for any chain of this family, $\gamma_{p s}$ is bounded from below by a universal constant. The Dobrushin coefficient $\mathcal{K}$ [defined at (2.8)] verifies

$$
\kappa\left(\boldsymbol{M}_{\sigma}\right)=\left\|\boldsymbol{\eta}(\boldsymbol{\sigma})-\boldsymbol{\eta}_{0}\right\|_{\mathrm{TV}}=8 \varepsilon \leq 1 / 2 .
$$

From the Bubley-Dyer path coupling method [Bubley and Dyer, 1997], $t_{\mathrm{mix}} \leq \frac{\ln 1 / 4}{\ln (1-\kappa)} \leq 2$, such that combining with Paulin [2015, Proposition 3.4], $\gamma_{\mathrm{ps}} \geq \frac{1}{2 t_{\operatorname{mix}}} \geq \frac{1}{4}$. A direct computation yields that for $\sigma \neq \sigma^{\prime},\left\|M_{\sigma}-M_{\sigma^{\prime}}\right\|_{1}=\frac{32 \varepsilon}{d} d_{H}\left(\sigma, \sigma^{\prime}\right)$, where $d_{H}$ is the Hamming distance. From the Varshamov-Gilbert lemma, we know that there is a $\Sigma \subset\{-1,1\}^{d / 2}$, $|\Sigma| \geq 2^{d / 16}$, such that for $\left(\sigma, \sigma^{\prime}\right) \in \Sigma$ with $\sigma \neq \sigma^{\prime}$, we have $d_{H}\left(\sigma, \sigma^{\prime}\right) \geq \frac{d}{16}$. Restricting our problem to this set $\Sigma$, and finally noticing that for $\sigma \in \Sigma$ we have $\left\|M_{\sigma}-M_{0}\right\|_{1}=16 \varepsilon>2 \varepsilon$, applying Tsybakov's method [Tsybakov, 2009, Theorem 2.5] to our problem, we obtain

$$
\mathcal{R}_{m} \geq \frac{1}{2}\left(1-\frac{\frac{4}{2^{\frac{d}{16}}} \sum_{\sigma \in \Sigma} \mathcal{D}_{m}}{\ln 2^{\frac{d}{16}}}\right)
$$

where $\mathcal{D}_{m}$ denotes the KL divergence between the two distributions of words of length $m$ (see formal definition at Lemma 6.2) from each of the Markov chains indexed by $\boldsymbol{\eta}(\sigma)$ and $\eta_{0}$. 
Leveraging the chain rule for the KL divergence, and as by construction, the only discrepancy occurs when visiting the $(d+1)$ th state, Lemma 6.2 shows the following tensorization property,

$$
\mathcal{D}_{m} \leq p_{\star} m D_{K L}\left(\boldsymbol{\eta}(\boldsymbol{\sigma}) \| \boldsymbol{\eta}_{0}\right),
$$

following up with a straightforward computation,

$$
D_{K L}\left(\boldsymbol{\eta}(\sigma) \| \boldsymbol{\eta}_{0}\right)=\sum_{s \in\{-1,+1\}} \frac{d}{2}\left(\frac{1-p_{\star}+16 s \varepsilon}{d}\right) \ln \left(\frac{\frac{1-p_{\star}+16 s \varepsilon}{d}}{\frac{1-p_{\star}}{d}}\right) \leq 128 \varepsilon^{2},
$$

and finally combining (6.4), (6.6) and (6.7), we get $\mathcal{R}_{m} \geq \frac{1}{2}\left(1-\frac{512 \varepsilon^{2} m p_{\star}}{\frac{d}{16} \ln 2}\right)$. Further noticing that for the considered range of $\varepsilon$ and for $p_{\star}<\frac{1}{d+2}$, it is always the case that $\pi_{\star}=p_{\star}$, so that for $m \leq \frac{d(1-2 \delta) \ln 2}{8192 \varepsilon^{2} \pi_{\star}}, \mathcal{R}_{m} \geq \delta$.

Lemma 6.2 For two Markov chains $\boldsymbol{M}_{1}$ and $\boldsymbol{M}_{2}$ of the class $\mathcal{G}_{p}$ defined at (6.5) indexed respectively by $\eta_{1}$ and $\eta_{2}$, denote respectively by $\mathcal{L}_{1}\left(\boldsymbol{X}_{1}^{m}\right)$ and $\mathcal{L}_{2}\left(\boldsymbol{X}_{2}^{m}\right)$ the distributions of words of length $m$, and write for simplicity $\mathcal{D}_{t}=D_{K L}\left(\mathcal{L}_{1}\left(\boldsymbol{X}_{1}^{t}\right)|| \mathcal{L}_{2}\left(\boldsymbol{X}_{2}^{t}\right)\right)$ the KL divergence between the processes up to time $t$. Then it is a fact that

$$
\mathcal{D}_{m}=(m-1) p_{\star} D_{K L}\left(\boldsymbol{\eta}_{1} \| \boldsymbol{\eta}_{2}\right) \text {. }
$$

Proof: From an application of the chain rule for the KL divergence, followed by the Markov property,

$$
\mathcal{D}_{m}=\mathcal{D}_{m-1}+\mathcal{E}_{m}
$$

where

$$
\begin{aligned}
\mathcal{E}_{m} & \doteq \mathbb{E}_{\boldsymbol{X}_{1}^{m-1} \sim \mathcal{L}_{1}}\left[\mathcal{D}_{m \mid m-1}\right], \\
\mathcal{D}_{m \mid m-1} & \doteq D_{K L}\left(\mathcal{L}_{1}\left(X_{m} \mid X_{m-1}\right)|| \mathcal{L}_{2}\left(X_{m} \mid X_{m-1}\right)\right) .
\end{aligned}
$$

In the event where $X_{m-1} \neq d+1, \mathcal{D}_{m \mid m-1}=0$, such that from the law of total expectation,

$$
\mathcal{E}_{m}=\mathbb{E}_{\boldsymbol{X}_{1}^{m-2} \sim \mathcal{L}_{1}}\left[\mathbb{E}\left[\mathcal{D}_{m \mid m-1} \mid X_{m-1}=d+1\right] \mathcal{L}_{1}\left(X_{m-1}=d+1 \mid \boldsymbol{X}_{1}^{m-2}\right)\right] .
$$

From a second application of the Markov property, and by structural property of the chain,

$$
\mathcal{L}_{1}\left(X_{m-1}=d+1 \mid X_{1}^{m-2}\right)=\mathcal{L}_{1}\left(X_{m-1}=d+1 \mid X_{m-2}\right)=M_{1}\left(X_{m-2}, d+1\right)=p_{\star},
$$

while in the event where $X_{m-1}=d+1$,

$$
\mathcal{D}_{m \mid m-1}=D_{K L}\left(\boldsymbol{\eta}_{1}|| \boldsymbol{\eta}_{2}\right) .
$$

Combining (6.8) with (6.9) and (6.10) yields,

$$
\mathcal{E}_{m}=p_{\star} D_{K L}\left(\boldsymbol{\eta}_{1}|| \eta_{2}\right) .
$$

From an inductive argument, and the base case $\mathcal{D}_{1}=0$,

$$
\mathcal{D}_{m}=(m-1) p_{\star} D_{K L}\left(\boldsymbol{\eta}_{1} \| \boldsymbol{\eta}_{2}\right) \text {. }
$$




\subsection{Proof of Theorem 3.2 (part 2): $\frac{d \ln d}{\gamma_{\mathrm{ps}}}$}

We treat $\varepsilon \in(0,1 / 8)$ and $d=6 k, k \geq 2$ as fixed. For $\eta \in(0,1 / 48)$ and $\tau \in\{0,1\}^{d / 3}$, define the block matrix

$$
M_{\eta, \tau}=\left(\begin{array}{ll}
C_{\eta} & R_{\tau} \\
R_{\tau}^{\dagger} & L_{\tau}
\end{array}\right)
$$

, where $C_{\eta} \in \mathbb{R}^{d / 3 \times d / 3}, L_{\tau} \in \mathbb{R}^{2 d / 3 \times 2 d / 3}$, and $\boldsymbol{R}_{\boldsymbol{\tau}} \in \mathbb{R}^{d / 3 \times 2 d / 3}$ are given by

$$
\begin{aligned}
& L_{\tau}=\frac{1}{8} \operatorname{diag}\left(7-4 \tau_{1} \varepsilon, 7+4 \tau_{1} \varepsilon, \ldots, 7-4 \tau_{d / 3} \varepsilon, 7+4 \tau_{d / 3} \varepsilon\right), \\
& C_{\eta}=\left(\begin{array}{cccc}
\frac{3}{4}-\eta & \frac{\eta}{d / 3-1} & \ldots & \frac{\eta}{d / 3-1} \\
\frac{\eta}{d / 3-1} & \frac{3}{4}-\eta & \ddots & \vdots \\
\vdots & \ddots & \ddots & \frac{\eta}{d / 3-1} \\
\frac{\eta}{d / 3-1} & \cdots & \frac{\eta}{d / 3-1} & \frac{3}{4}-\eta
\end{array}\right) \\
& \boldsymbol{R}_{\tau}=\frac{1}{8}\left(\begin{array}{ccccccc}
1+4 \tau_{1} \varepsilon & 1-4 \tau_{1} \varepsilon & 0 & \ldots & \ldots & \ldots & 0 \\
0 & 0 & 1+4 \tau_{2} \varepsilon & 1-4 \tau_{2} \varepsilon & 0 & \ldots & 0 \\
\vdots & \vdots & \vdots & \vdots & \vdots & \vdots & \vdots \\
0 & \ldots & \ldots & \ldots & 0 & 1+4 \tau_{d / 3} \varepsilon & 1-4 \tau_{d / 3} \varepsilon
\end{array}\right)
\end{aligned}
$$

Holding $\eta$ fixed, define the collection

$$
\mathcal{H}_{\eta}=\left\{\boldsymbol{M}_{\eta, \tau}: \tau \in\{0,1\}^{d / 3}\right\}
$$

of Markov matrices. Denote by $\boldsymbol{M}_{\eta, 0} \in \mathcal{H}_{\eta}$ the element corresponding to $\boldsymbol{\tau}=\mathbf{0}$. Note that every $\boldsymbol{M} \in \mathcal{H}_{\eta}$ is ergodic and reversible, and its unique stationary distribution is uniform. A graphical illustration of this class of Markov chains is provided in Figure 1; in particular, every $\boldsymbol{M} \in \mathcal{H}_{\eta}$ consists of an "inner clique" (i.e., the states indexed by $\{1, \ldots, d / 3\}$ ) and "outer rim" (i.e., the states indexed by $\{d / 3+1, \ldots, d\}$ ).

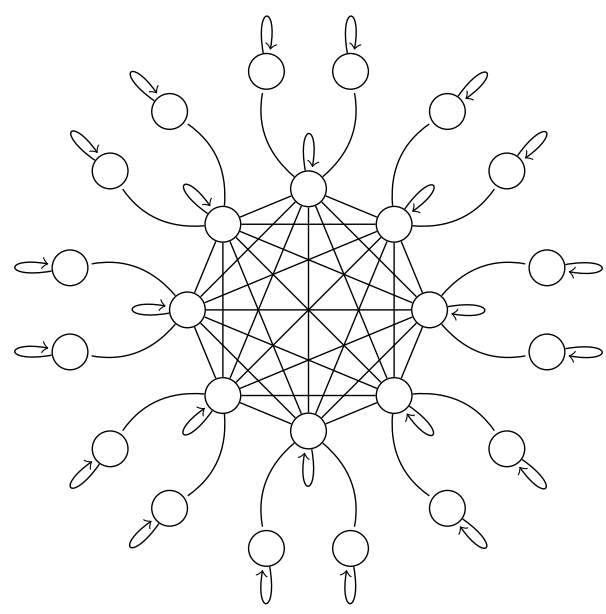

Figure 1: Generic topology of the $\mathcal{H}_{\eta}$ Markov chain class: every chain consists of an "inner clique" and an "outer rim". 
Lemma 6.3 in the Appendix establishes a key property of the elements of $\mathcal{H}_{\eta}$ : each $\boldsymbol{M}$ in this class satisfies

$$
\eta / 4 \leq \gamma_{\mathrm{ps}} \leq \eta
$$

Suppose that $\boldsymbol{X}=\left(X_{1}, \ldots, X_{m}\right) \sim\left(M_{\eta}, \pi\right)$, where $\boldsymbol{M} \in \mathcal{H}_{\eta}$ and $\pi$ is uniform. Define the random variable $T_{\text {CLIQ }}$, to be the first time all of the states in the inner clique were visited,

$$
T_{\text {CLIQ }}=\inf \left\{t \geq 1:\left|\left\{X_{1}, \ldots, X_{t}\right\} \cap[d / 3]\right|=d / 3\right\},
$$

Lemma 6.4 in the Appendix gives a lower estimate on this quantity:

$$
m \leq \frac{d}{20 \eta} \ln \left(\frac{d}{3}\right) \Longrightarrow \mathbb{P}\left(T_{\text {CLI }}>m\right) \geq \frac{1}{5} \text {. }
$$

Let $\mathcal{M}_{d, \gamma_{\mathrm{ps}}, \pi_{\star}}$ be the collection of all $d$-state Markov chains whose stationary distribution is minorized by $\pi_{\star}$ and whose pseudo-spectral gap is at least $\gamma_{\mathrm{ps}}$. Writing $\boldsymbol{X}=\left(X_{1}, \ldots, X_{m}\right)$, recall that the quantity we wish to lower bound is the minimax risk for the statistical estimation problem (it will be convenient to write $\varepsilon / 2$ instead of $\varepsilon$, which only affects the constants):

$$
\mathcal{R}_{m}=\inf _{\widehat{\boldsymbol{M}}} \sup _{\boldsymbol{M}} \mathbb{P}_{\boldsymbol{M}}\left(\|\boldsymbol{M}-\widehat{\boldsymbol{M}}\|_{\infty}>\frac{\varepsilon}{2}\right)
$$

where the inf is taken over all estimation procedures $\widehat{\boldsymbol{M}}: \Omega^{m} \rightarrow \mathcal{M}_{d}, \boldsymbol{X} \mapsto \widehat{\boldsymbol{M}}(\boldsymbol{X})$, and the sup over $\mathcal{M}_{d, \gamma_{\mathrm{ps}}, \pi_{\star}}$. We employ the general reduction scheme of Tsybakov [2009, Chapter 2.2]. The first step is to restrict the sup to the finite subset $\mathcal{H}_{\eta} \subsetneq \mathcal{M}_{d, \gamma_{\mathrm{ps}}, \pi_{\star}}$.

$$
\mathcal{R}_{m} \geq \inf _{\widehat{M}} \max _{\tau} \mathbb{P}_{\boldsymbol{M}_{\eta, \tau}}\left(\left\|\boldsymbol{M}_{\eta, \tau}-\widehat{\boldsymbol{M}}\right\|_{\infty}>\frac{\varepsilon}{2}\right)
$$

Define $T_{\text {CLIQ }}$ as in (6.12). Then

$$
\mathcal{R}_{m} \geq \inf _{\widehat{\boldsymbol{M}}} \max _{\boldsymbol{\tau}} \mathbb{P}_{\boldsymbol{M}_{\eta, \tau}}\left(\left\|\boldsymbol{M}_{\eta, \boldsymbol{\tau}}-\widehat{\boldsymbol{M}}\right\|_{\infty}>\varepsilon \mid T_{\text {CLIQ }}>m\right) \mathbb{P}_{\boldsymbol{M}_{\eta, \tau}}\left(T_{\text {CLIQ }}>m\right)
$$

and Lemma 6.4 implies that for $m<\frac{d}{20 \eta} \ln \left(\frac{d}{3}\right)$,

$$
\mathcal{R}_{m} \geq \frac{1}{5} \inf _{\widehat{\boldsymbol{M}}} \max _{\boldsymbol{\tau}} \mathbb{P}_{\boldsymbol{M}_{\eta, \tau}}\left(\left\|\boldsymbol{M}_{\eta, \boldsymbol{\tau}}-\widehat{\boldsymbol{M}}\right\|_{\infty}>\varepsilon \mid T_{\mathrm{CLIQ}}>m\right) .
$$

Observe that all $\boldsymbol{\tau} \neq \boldsymbol{\tau}^{\prime} \in\{0,1\}^{d / 3}$ verify $\left\|\boldsymbol{M}_{\eta, \boldsymbol{\tau}}-\boldsymbol{M}_{\eta, \boldsymbol{\tau}^{\prime}}\right\|_{\infty}=\varepsilon$. For any estimate $\widehat{\boldsymbol{M}}$, define

$$
\tau^{\star}=\underset{\tau}{\operatorname{argmin}}\left\|\widehat{M}-M_{\eta, \tau}\right\|_{\infty} .
$$

Then for $\tau \neq \tau^{\star}$, we have

$$
\varepsilon=\left\|\boldsymbol{M}_{\eta, \tau}-\boldsymbol{M}_{\eta, \tau^{\star}}\right\|_{\infty} \leq\left\|\boldsymbol{M}_{\eta, \tau}-\widehat{\boldsymbol{M}}\right\|_{\infty}+\left\|\widehat{\boldsymbol{M}}-\boldsymbol{M}_{\eta, \tau^{\star}}\right\|_{\infty} \leq 2\left\|\boldsymbol{M}_{\eta, \tau}-\widehat{\boldsymbol{M}}\right\|_{\infty},
$$

whence $\left\{\boldsymbol{\tau}^{\star} \neq \boldsymbol{\tau}\right\} \subset\left\{\left\|\boldsymbol{M}_{\eta, \tau}-\widehat{\boldsymbol{M}}\right\|_{\infty}>\varepsilon / 2\right\}$ and

$$
\begin{aligned}
\mathcal{R}_{m} & \geq \frac{1}{5} \inf _{\widehat{\boldsymbol{M}}} \max _{\boldsymbol{\tau}} \mathbb{P}_{\boldsymbol{M}_{\eta, \boldsymbol{\tau}}}\left(\boldsymbol{\tau}^{\star} \neq \boldsymbol{\tau} \mid T_{\text {CLIQ }}>m\right) \\
& =\frac{1}{5} \inf _{\hat{\boldsymbol{\tau}}: \boldsymbol{X} \mapsto\{0,1\}^{d / 3}} \sup _{\boldsymbol{\tau}} \mathbb{P}_{\boldsymbol{M}_{\eta, \boldsymbol{\tau}}}\left(\hat{\boldsymbol{\tau}} \neq \boldsymbol{\tau} \mid T_{\text {CLIQ }}>m\right) .
\end{aligned}
$$


Since $T_{\text {CLIQ }}>m$ implies that $N_{i}=0$ for some $i \in[d / 3]$,

$$
\mathcal{R}_{m} \geq \frac{1}{5} \inf _{\hat{\tau}} \sup _{\boldsymbol{\tau}} \mathbb{P}_{\boldsymbol{M}_{\eta, \tau}}\left(\hat{\tau}_{i} \neq \tau_{i} \mid N_{i}=0\right) \text {. }
$$

There are as many $\boldsymbol{M} \in \mathcal{H}_{\eta}$ with $\tau_{i}=0$ as those with $\tau_{i}=1$, so if $\boldsymbol{M}$ is drawn uniformly at random and state $i$ has not been visited, one can do no better than to make a random choice of $\hat{\tau}_{i}$ (where $\hat{\boldsymbol{\tau}}$ determines $\widehat{\boldsymbol{M}}$ ). More formally, writing $\boldsymbol{\tau}^{(i)}=\left(\tau_{1}, \ldots, \tau_{i-1}, \tau_{i+1}, \ldots, \tau_{d / 3}\right) \in$ $\{0,1\}^{d / 3-1}$, the $\tau$ vector without its $i$ th coordinate, we can employ an Assouad-type of decomposition [Assouad, 1983, Yu, 1997]:

$$
\begin{aligned}
\mathcal{R}_{m} & \geq \frac{1}{5} \inf _{\hat{\tau}} 2^{1-d / 3} \sum_{\tau^{(i)} \in\{0,1\}^{d / 3-1}}\left[\frac{1}{2} \mathbb{P}_{\tau_{i}=0}\left(\hat{\tau}_{i} \neq \tau_{i} \mid N_{i}=0\right)+\frac{1}{2} \mathbb{P}_{\tau_{i}=1}\left(\hat{\tau}_{i} \neq \tau_{i} \mid N_{i}=0\right)\right] \\
& =\frac{2^{1-d / 3}}{10} \sum_{\tau^{(i)} \in\{0,1\}^{d / 3-1}} \inf _{\hat{\tau}}\left[\mathbb{P}_{\tau_{i}=0}\left(\hat{\tau}_{i}=1 \mid N_{i}=0\right)+\mathbb{P}_{\tau_{i}=1}\left(\hat{\tau}_{i}=0 \mid N_{i}=0\right)\right] \\
& =\frac{2^{1-d / 3}}{10} \sum_{\tau^{(i)} \in\{0,1\}^{d / 3-1}}\left[1-\left\|\mathbb{P}_{\tau_{i}=0}\left(X=\cdot \mid N_{i}=0\right)+\mathbb{P}_{\tau_{i}=1}\left(X=\cdot \mid N_{i}=0\right)\right\|_{\mathrm{TV}}\right] \\
& =\frac{1}{10} .
\end{aligned}
$$

Combined with Lemma 6.3, and inclusion of events, this implies lower bound of $\frac{d}{\gamma_{\mathrm{ps}}} \ln d$ for the estimation problem, which is tight for the case $\pi_{\star}=\frac{1}{d}$.

Remark 6.3 Let us compare construction $\mathcal{H}_{\eta}$ to the family of Markov chains employed in the lower bound of Hsu et al. [2019]:

$$
\boldsymbol{M}(i, j)= \begin{cases}1-\eta_{i}, & i=j \\ \frac{\eta_{i}}{d-1}, & \text { else }\end{cases}
$$

where $\eta_{i} \in\left\{\eta, \eta^{\prime}\right\}$ with $\eta^{\prime} \approx \eta / 2$. For our lower bound, $\mathcal{H}_{\eta}^{\prime}$ has to be a $\varepsilon$-separated set under $\|\cdot\|_{\infty}$. In the construction of Hsu et al., the spectral gap $\gamma$ and the separation distance $\varepsilon$ are coupled, and using their family of Markov chains would lead to a lower bound of order $d / \gamma \approx d / \varepsilon$, which is inferior to $\frac{d}{\varepsilon^{2} \pi_{\star}}$. The free parameter $\eta$ was key to our construction, which enabled us to decouple $\gamma$ from $\varepsilon$.

Lemma 6.3 Let $\varepsilon \in(0,1 / 32)$ and $\eta \in(0,1 / 48)$. For all $\boldsymbol{M} \in \mathcal{H}_{\eta}$ [defined in (6.11)], we have $\eta / 4 \leq \gamma_{\star} \leq \gamma_{\mathrm{ps}} \leq \eta$.

Proof: We focus our proof on the absolute spectral gap, and will later show that the pseudo spectral gap is of the same order for our class of Markov matrices. A lower bound for $\gamma_{\star}$ of the unperturbed chain $\boldsymbol{M}_{\eta, 0}$, is given by Lemma 6.5. We now show how to extend to general $\boldsymbol{\tau}$ with comparison techniques. It is well known that (see i.e. Levin et al. [2009, Lemma 13.7]) that for a reversible chain $\boldsymbol{M}$,

$$
\gamma(\boldsymbol{M})=\min _{\substack{f:[d] \rightarrow \mathbb{R} \\ f \perp_{\pi} \mathbf{1},\|f\|_{2}=1}} \mathcal{E}_{\boldsymbol{M}}(f)
$$


where

$$
\mathcal{E}_{\boldsymbol{M}}(f) \doteq \frac{1}{2} \sum_{(i, j) \in[d]^{2}}(f(i)-f(j))^{2} \boldsymbol{\pi}(i) \boldsymbol{M}(i, j)
$$

is the Dirichlet form associated to $M$ with stationary distribution $\pi$. We now use this variational definition to control the spectral gap of the perturbed chains $\boldsymbol{M}_{\eta, \tau}$ in terms of the one of $\boldsymbol{M}_{\eta, 0}$, relying on the fact that for both chains, the stationary distribution is uniform. Comparing transition matrices,

$$
\begin{aligned}
\mathcal{E}_{\boldsymbol{M}_{\eta, \tau}}(f) & =\frac{1}{2} \sum_{(i, j) \in[d]^{2}}(f(i)-f(j))^{2} \boldsymbol{\pi}(i) \boldsymbol{M}_{\eta, \boldsymbol{\tau}}(i, j) \\
& \geq \frac{1}{2} \sum_{(i, j) \in[d]^{2}}(f(i)-f(j))^{2}(1 / d)(1-4 \varepsilon) \boldsymbol{M}_{\eta, \mathbf{0}}(i, j) \\
& =(1-4 \varepsilon) \mathcal{E}_{\boldsymbol{M}_{\eta, 0}}(f),
\end{aligned}
$$

and by the definition at (6.13), $\gamma\left(\boldsymbol{M}_{\eta, \boldsymbol{\tau}}\right) \geq(1-4 \varepsilon) \gamma\left(\boldsymbol{M}_{\eta, 0}\right)$.

Extension to $\gamma_{\mathrm{ps}}$. Now note that for a symmetric and lazy $\boldsymbol{M}, \boldsymbol{\pi}$ is the uniform distribution, $\boldsymbol{M}^{\dagger}=\boldsymbol{M}^{\boldsymbol{\top}}=\boldsymbol{M}$, and $\gamma_{\mathrm{ps}}=\max _{k \geq 1}\left\{\frac{\gamma\left(\boldsymbol{M}^{2 k}\right)}{k}\right\}$. Denoting by $1=\lambda_{1}>\lambda_{2} \geq \cdots \geq \lambda_{d}$ the eigenvalues of $\boldsymbol{M}$, we have that for all $i \in[d]$ and $k \geq 1, \lambda_{i}^{2 k}$ is an eigenvalue for $\boldsymbol{M}^{2 k}$, and furthermore $1=\lambda_{1}^{2 k}>\lambda_{2}^{2 k} \geq \cdots \geq \lambda_{d}^{2 k}$. Then

$$
\gamma_{\mathrm{ps}}=\max _{k \geq 1} \frac{1-\lambda_{2}^{2 k}}{k}=1-\lambda_{2}^{2}
$$

- that is, the maximum is achieved at $k=1$. Indeed, $1-\lambda_{2}^{2 k}=\left(1-\lambda_{2}^{2}\right)\left(\sum_{i=0}^{k-1} \lambda_{2}^{2 i}\right)$ and the latter sum is at most $k$ since $\lambda_{2}^{2}<\lambda_{2}<1$. As a result, $\gamma_{\mathrm{ps}}=1-\lambda_{2}^{2}=1-(1-\gamma)^{2}=$ $\gamma(2-\gamma)$ and

$$
\gamma \leq \gamma_{\mathrm{ps}} \leq 2 \gamma
$$

which completes the proof.

Lemma 6.4 (Cover time) For $\boldsymbol{M} \in \mathcal{H}_{\eta}\left[\right.$ defined in (6.11)], the random variable $T_{\text {CLI }}[$ defined in (6.12)] satisfies

$$
m \leq \frac{d}{20 \eta} \ln \left(\frac{d}{3}\right) \Longrightarrow \mathbb{P}\left(T_{\text {CLIQ }}>m\right) \geq \frac{1}{5}
$$

Proof: Let $\boldsymbol{M} \in \mathcal{H}_{\eta}$ and $\boldsymbol{M}_{I} \in \mathcal{M}_{d / 3}$ be such that $\boldsymbol{M}_{I}$ consists only in the inner clique of $\boldsymbol{M}$, and each outer rim state got absorbed into its unique inner clique neighbor:

$$
\mathbf{M}_{I}=\left(\begin{array}{cccc}
1-\eta & \frac{\eta}{d / 3-1} & \ldots & \frac{\eta}{d / 3-1} \\
\frac{\eta}{d / 3-1} & 1-\eta & \ddots & \vdots \\
\vdots & \ddots & \ddots & \frac{\eta}{d / 3-1} \\
\frac{\eta}{d / 3-1} & \ldots & \frac{\eta}{d / 3-1} & 1-\eta
\end{array}\right)
$$


By construction, it is clear that $T_{\text {CLI }}$ is almost surely greater than the cover time of $\boldsymbol{M}_{I}$. The latter corresponds to a generalized coupon collection time $U=1+\sum_{i=1}^{d / 3-1} U_{i}$ where $U_{i}$ is the time increment between the $i$ th and the $(i+1)$ th unique visited state. Formally, if $\boldsymbol{X}$ is a random walk according to $M_{I}$ (started from any state), then $U_{1}=\min \left\{t>1: X_{t} \neq X_{1}\right\}$ and for $i>1$,

$$
U_{i}=\min \left\{t>1: X_{t} \notin\left\{X_{1}, \ldots, X_{U_{i-1}}\right\}\right\}-U_{i-1} .
$$

The random variables $U_{1}, U_{2}, \ldots, U_{d / 3-1}$ are independent and

$$
U_{i} \sim \text { Geometric }\left(\eta-\frac{(i-1) \eta}{d / 3}\right)
$$

whence

$$
\mathbb{E}\left[U_{i}\right]=\frac{d / 3}{\eta(d / 3-i+1)^{\prime}}, \quad \operatorname{Var}\left[U_{i}\right]=\left(1-\left(\eta-\frac{(i-1) \eta}{d / 3}\right)\right)\left(\eta-\frac{(i-1) \eta}{d / 3}\right)^{-2}
$$

and

$$
\mathbb{E}[U] \geq 1+\frac{d / 3}{\eta} \sigma_{d / 3-1}, \quad \mathbb{V a r}[U] \leq \frac{(d / 3-1)^{2}}{\eta^{2}} \frac{\pi^{2}}{6}
$$

where $\sigma_{d}=\sum_{i=1}^{d} \frac{1}{i}$, and $\pi=3.1416 \ldots$ Invoking the Paley-Zygmund inequality with $\theta=$ $1-\frac{2 \sqrt{2 / 3}}{\sigma_{d / 3-1}}$ we have

$$
\mathbb{P}(U>\theta \mathbb{E}[U]) \geq\left(1+\frac{\mathbb{V a r}[U]}{(1-\theta)^{2}(\mathbb{E}[U])^{2}}\right)^{-1} \geq \frac{1}{5}
$$

Further, $\sigma_{d / 3-1} \geq \sigma_{3}=11 / 6$ implies

$$
\theta \mathbb{E}[U] \geq \frac{3}{20} \cdot \frac{d / 3}{\eta} \sigma_{d / 3-1} \geq \frac{d}{20 \eta} \ln \left(\frac{d}{3}\right)
$$

and thus for $m \leq \frac{d}{20 \eta} \ln \left(\frac{d}{3}\right)$, we have $\mathbb{P}\left(T_{\text {CLI }}>m\right) \geq \frac{1}{5}$.

Lemma 6.5 (Spectrum of $\boldsymbol{M}_{\eta, 0} \in \mathcal{H}_{\eta}$ ) Let $d=6 k, k \geq 2$ and $0<\eta<1 / 48$, and write $c_{d} \doteq d /(d-3)$. The spectrum of $\boldsymbol{M}_{\eta, 0}$ is

$$
\operatorname{Spec}\left(\boldsymbol{M}_{\eta, 0}\right)=\left\{\lambda_{1}, \lambda_{+}, \bar{\lambda}, \underline{\lambda}, \lambda_{-}\right\}
$$

where $\lambda_{1}=1$ (mult. 1$), \lambda_{ \pm} \doteq \frac{1}{16}\left(13-8 \eta c_{d} \pm \sqrt{\left(3+8 \eta c_{d} / 3\right)^{2}+512 \eta^{2} c_{d}^{2} / 9}\right)$ (each mult. $d / 3-1), \underline{\lambda}=5 / 8$ (mult. 1$), \bar{\lambda}=7 / 8($ mult. $d / 3)$.

Moreover, $\lambda_{\star} \doteq \max _{\lambda \in \operatorname{Spec}\left(M_{\eta, 0}\right)}\{|\lambda|, \lambda \neq 1\}=\lambda_{+}$, and $\eta / 4 \leq \gamma_{\star} \leq \eta / 2$. 
Proof: $\quad$ By definition, and writing $c_{d} \doteq d /(d-3), \boldsymbol{M}_{\eta, 0}=\left(\begin{array}{ll}C_{\eta} & \boldsymbol{R}_{\mathbf{0}} \\ \boldsymbol{R}_{\mathbf{0}}^{\top} & \boldsymbol{L}_{\mathbf{0}}\end{array}\right)$, where $\boldsymbol{L}_{\mathbf{0}}=\frac{7}{8} \boldsymbol{I}$,

$$
\boldsymbol{C}_{\eta}=\frac{\eta}{d / 3-1} \mathbf{1}^{\top} \cdot \mathbf{1}-\left(\eta c_{d}-\frac{3}{4}\right) \boldsymbol{I} \text { and } \boldsymbol{R}_{\mathbf{0}}=\frac{1}{8}\left(\begin{array}{ccccccc}
1 & 1 & 0 & \ldots & \ldots & \ldots & 0 \\
0 & 0 & 1 & 1 & 0 & \ldots & 0 \\
\vdots & \vdots & \vdots & \vdots & \vdots & \vdots & \vdots \\
0 & \ldots & \ldots & \ldots & 0 & 1 & 1
\end{array}\right) .
$$

As $\boldsymbol{M}_{\eta, 0}$ is a symmetric matrix, its spectrum is real. Let $\lambda \in \mathbb{R}$, and suppose first that $\lambda \neq 7 / 8$. In this case, $\left|\boldsymbol{L}_{\mathbf{0}}-\lambda \boldsymbol{I}\right| \neq 0$, and leveraging the block-structure of the matrix, it is a classical result (see for example Silvester [2000]) that

$$
\left|\boldsymbol{M}_{\eta, \mathbf{0}}-\lambda \boldsymbol{I}\right|=\left|\boldsymbol{L}_{\mathbf{0}}-\lambda \boldsymbol{I}\right| \cdot\left|\boldsymbol{C}_{\eta}-\lambda \boldsymbol{I}-\boldsymbol{R}_{\mathbf{0}}\left(\boldsymbol{L}_{\mathbf{0}}-\lambda \boldsymbol{I}\right)^{-1} \boldsymbol{R}_{\mathbf{0}}^{\top}\right| \cdot
$$

A direct computation shows that

$$
\boldsymbol{R}_{\mathbf{0}}\left(\boldsymbol{L}_{\mathbf{0}}-\lambda \boldsymbol{I}\right)^{-1} \boldsymbol{R}_{\mathbf{0}}^{\top}=\frac{1}{4(7-8 \lambda)} \boldsymbol{I},
$$

such that,

$$
\boldsymbol{C}_{\eta}-\lambda \boldsymbol{I}-\boldsymbol{R}_{\mathbf{0}}\left(\boldsymbol{L}_{\mathbf{0}}-\lambda \boldsymbol{I}\right)^{-1} \boldsymbol{R}_{\mathbf{0}}^{\boldsymbol{\top}}=\frac{\eta}{d / 3-1} \mathbf{1}^{\boldsymbol{\top}} \cdot \mathbf{1}-\left(\eta c_{d}-\frac{3}{4}+\lambda+\frac{1}{4(7-8 \lambda)}\right) \boldsymbol{I} .
$$

This implies that $\left|\boldsymbol{C}_{\eta}-\lambda \boldsymbol{I}-\boldsymbol{R}_{\mathbf{0}}\left(\boldsymbol{L}_{\mathbf{0}}-\lambda \boldsymbol{I}\right)^{-1} \boldsymbol{R}_{\mathbf{0}}^{\top}\right|=0$ if and only if, $\eta c_{d}-\frac{3}{4}+\lambda+\frac{1}{4(7-8 \lambda)} \in$ $\operatorname{Spec}\left(\eta /(d / 3-1) \mathbf{1}^{\boldsymbol{\top}} \cdot \mathbf{1}\right)=\left\{0, \eta c_{d}\right\}$ where 0 has multiplicity $d / 3-1$ and $\eta c_{d}$ has multiplicity 1 . Let $\xi \geq 0$, then solutions for the equation $\xi+\lambda+\frac{1}{4(7-8 \lambda)}=\frac{3}{4}$ are given by

$$
\lambda_{ \pm}(\xi)=\frac{13-8 \xi \pm \sqrt{(3+8 \xi / 3)^{2}+512 \xi^{2} / 9}}{16}
$$

Setting $\xi=0$ yields that $\lambda_{1}=1$ and $\underline{\lambda}=5 / 8$ are eigenvalues both with multiplicity 1 , while setting $\xi=\eta c_{d}$ yields that

$$
\lambda_{ \pm}=\frac{13-8 \eta c_{d} \pm \sqrt{\left(3+8 \eta c_{d} / 3\right)^{2}+512 \eta^{2} c_{d}^{2} / 9}}{16}
$$

are both eigenvalues with multiplicity $d / 3-1$. As the characteristic polynomial of $\boldsymbol{M}_{\eta, 0}$ has degree $d$, a natural consequence is that $\bar{\lambda}=7 / 8$ is another eigenvalue with multiplicity $d / 3$. It remains to order $\lambda_{1}, \lambda_{-}, \lambda_{+}, \underline{\lambda}, \bar{\lambda}$. Since $\boldsymbol{M}_{\eta, 0}$ is lazy, all eigenvalues are positive. Trivially, $\lambda_{1}$ is the largest eigenvalue, $\bar{\lambda}>\underline{\lambda}$ and always $\lambda_{-} \leq \lambda_{+}$. Additionally, $512 \eta^{2} c_{d}^{2} / 9 \geq 0$ implies that $\lambda_{+} \geq 1-\eta c_{d} / 3 \geq 1-\eta / 2$ for the considered range of $d$, which is in turn larger than $7 / 8$ for $\eta \leq 1 / 4$. As a result $\lambda_{\star}=\lambda_{+}$and $\gamma_{\star} \leq \eta / 2$. Furthermore, as one can write $\lambda_{+}=\frac{1}{16}\left(13-8 \eta c_{d} \pm \sqrt{\left(3+4 \eta c_{d} / 3\right)^{2}-8 c_{d} \eta\left(1-8 c_{d} \eta\right)}\right)$, and since $1-8 c_{d} \eta \geq 0$, $\lambda_{+} \leq 1-\eta / 4$, whence $\gamma_{\star} \geq \eta / 4$.

\section{Acknowledgments}

We are thankful to John Lafferty for bringing this problem to our attention and numerous insightful conversations. We also thank the anonymous referees, who made valuable comments and suggestions, including shaving off a logarithmic factor in Theorem 3.1 and the explicit computation of the eigenvalues in Lemma 6.5. This research was partially supported by the Israel Science Foundation (grant No. 755/15), Paypal and IBM. 


\section{References}

M. Anthony and P. L. Bartlett. Neural Network Learning: Theoretical Foundations. Cambridge University Press, Cambridge, 1999. ISBN 0-521-57353-X. doi: 10.1017/ CBO9780511624216. URL http://dx.doi.org/10.1017/CB09780511624216.

P. Assouad. Deux remarques sur l'estimation. Comptes rendus des séances de l'Académie des sciences. Série 1, Mathématique, 296(23):1021-1024, 1983.

D. Berend and A. Kontorovich. A sharp estimate of the binomial mean absolute deviation with applications. Statistics \& Probability Letters, 83(4):1254-1259, 2013.

P. Billingsley. Statistical methods in markov chains. The Annals of Mathematical Statistics, 32(1):12-40, 1961. ISSN 00034851. URL http://www.jstor.org/stable/ 2237603.

R. Bubley and M. Dyer. Path coupling: A technique for proving rapid mixing in markov chains. In Proceedings 38th Annual Symposium on Foundations of Computer Science, pages 223-231. IEEE, 1997.

B. A. Craig and P. P. Sendi. Estimation of the transition matrix of a discrete-time Markov chain. Health economics, 11(1):33-42, 2002.

L. Devroye and G. Lugosi. Combinatorial methods in density estimation. Springer Series in Statistics. Springer-Verlag, New York, 2001. ISBN 0-387-951172. doi: 10.1007/978-1-4613-0125-7. URL http://dx.doi.org/10.1007/ $978-1-4613-0125-7$.

J. A. Fill. Eigenvalue bounds on convergence to stationarity for nonreversible Markov chains, with an application to the exclusion process. Ann. Appl. Probab., 1(1):62-87, 1991. doi: 10.1214/aoap/1177005981.

Y. Han, J. Jiao, and T. Weissman. Minimax estimation of discrete distributions under $\ell_{1}$ loss. IEEE Transactions on Information Theory, 61(11):6343-6354, Nov 2015. ISSN 0018-9448. doi: 10.1109/TIT.2015.2478816.

Y. Hao, A. Orlitsky, and V. Pichapati. On learning markov chains. In Advances in Neural Information Processing Systems, pages 646-655, 2018.

R. A. Horn and C. R. Johnson. Matrix Analysis. Cambridge University Press, 1985.

D. Hsu, A. Kontorovich, D. A. Levin, Y. Peres, C. Szepesvári, and G. Wolfer. Mixing time estimation in reversible markov chains from a single sample path. Ann. Appl. Probab., 29 (4):2439-2480, 08 2019. doi: 10.1214/18-AAP1457. URL https://doi.org/10. $1214 / 18-A A P 1457$.

S. Kamath, A. Orlitsky, D. Pichapati, and A. T. Suresh. On learning distributions from their samples. In Proceedings of The 28th Conference on Learning Theory, COLT 2015, Paris, France, July 3-6, 2015, pages 1066-1100, 2015. URL http://jmlr.org/ proceedings/papers/v40/Kamath15.html. 
M. J. Kearns, Y. Mansour, D. Ron, R. Rubinfeld, R. E. Schapire, and L. Sellie. On the learnability of discrete distributions. In Proceedings of the Twenty-Sixth Annual ACM Symposium on Theory of Computing, 23-25 May 1994, Montréal, Québec, Canada, pages 273282, 1994. doi: 10.1145/195058.195155. URL http://doi.acm.org/10.1145/ 195058.195155.

J. G. Kemeny and J. L. Snell. Finite Markov chains. Springer-Verlag, New York, 1976. Reprinting of the 1960 original, Undergraduate Texts in Mathematics.

A. Kontorovich and I. Pinelis. Exact lower bounds for the agnostic probably-approximatelycorrect (pac) machine learning model. Ann. Statist., 47(5):2822-2854, 2019. ISSN 00905364. doi: 10.1214/18-AOS1766.

A. L. Kontorovich. Measure Concentration of Strongly Mixing Processes with Applications. $\mathrm{PhD}$ thesis, Carnegie Mellon University, 2007.

L. A. Kontorovich and K. Ramanan. Concentration Inequalities for Dependent Random Variables via the Martingale Method. Ann. Probab., 36(6):2126-2158, 2008.

D. A. Levin, Y. Peres, and E. L. Wilmer. Markov chains and mixing times, second edition. American Mathematical Soc., 2009.

A. A. Markov. Extension of the law of large numbers to dependent quantities. Izvestiia Fiz.Matem. Obsch. Kazan Univ., 15:135-156, 1906.

A. Orlitsky and A. T. Suresh. Competitive distribution estimation: Why is good-turing good. In Advances in Neural Information Processing Systems 28: Annual Conference on Neural Information Processing Systems 2015, December 7-12, 2015, Montreal, Quebec, Canada, pages 2143-2151, 2015. URL http://papers.nips.cc/paper/ 5762-competitive-distribution-estimation-why-is-good-turing-good.

D. Paulin. Concentration inequalities for Markov chains by Marton couplings and spectral methods. Electronic Journal of Probability, 20, 2015.

G. Roberts, J. Rosenthal, et al. Geometric ergodicity and hybrid markov chains. Electronic Communications in Probability, 2:13-25, 1997.

J. R. Silvester. Determinants of block matrices. The Mathematical Gazette, 84(501):460-467, 2000. ISSN 00255572. URL http: / / www.jstor.org/stable/3620776.

A. B. Tsybakov. Introduction to nonparametric estimation, 2009. URL https: / / doi . org / 10.1007/b13794. Revised and extended from the 2004 French original, Translated by Vladimir Zaiats.

M. Vidyasagar. An elementary derivation of the large deviation rate function for finite state $\mathrm{m}$ arkov chains. Asian Journal of Control, 16(1):1-19, 2014.

B. Waggoner. $L_{p}$ testing and learning of discrete distributions. In Proceedings of the 2015 Conference on Innovations in Theoretical Computer Science, ITCS 2015, Rehovot, Israel, January 11-13, 2015, pages 347-356, 2015. doi: 10.1145/2688073.2688095. URL http: //doi.acm.org/10.1145/2688073.2688095. 
N. Welton and A. E. Ades. Estimation of Markov chain transition probabilities and rates from fully and partially observed data: uncertainty propagation, evidence synthesis, and model calibration. Medical Decision Making, 25(6):633-645, 2005.

G. Wolfer. Mixing time estimation in ergodic markov chains from a single trajectory with contraction methods. In A. Kontorovich and G. Neu, editors, Proceedings of the 31st International Conference on Algorithmic Learning Theory, volume 117 of Proceedings of Machine Learning Research, pages 890-905, San Diego, California, USA, 08 Feb-11 Feb 2020. PMLR. URL http://proceedings.mlr.press/v117/wolfer20a.html.

G. Wolfer and A. Kontorovich. Minimax learning of ergodic markov chains. In Proceedings of the 30th International Conference on Algorithmic Learning Theory, volume 98 of Proceedings of Machine Learning Research, pages 904-930, Chicago, Illinois, 22-24 Mar 2019a. PMLR. URL http: //proceedings.mlr.press/v98/wolfer19a.html.

G. Wolfer and A. Kontorovich. Estimating the mixing time of ergodic markov chains. In Proceedings of the Thirty-Second Conference on Learning Theory, volume 99 of Proceedings of Machine Learning Research, pages 3120-3159, Phoenix, USA, 25-28 Jun 2019b. PMLR. URL http: / / proceedings.mlr.press/v99/wolfer19a.html.

B. Yu. Assouad, Fano, and Le Cam. In Festschrift for Lucien Le Cam, pages 423-435. Springer, 1997. 